\title{
USP25 regulates Wnt signaling by controlling the stability of tankyrases
}

\author{
Daichao Xu, ${ }^{1,2,4}$ Jianping Liu, ${ }^{3,4}$ Tao Fu, ${ }^{3}$ Bing Shan, ${ }^{1}$ Lihui Qian, ${ }^{1}$ Lifeng Pan, ${ }^{3}$ and Junying Yuan ${ }^{1,2}$ \\ ${ }^{1}$ Interdisciplinary Research Center on Biology and Chemistry, Shanghai Institute of Organic Chemistry, Chinese Academy \\ of Sciences, Pudong, Shanghai 201210, China; ${ }^{2}$ Department of Cell Biology, Harvard Medical School, Boston, Massachusetts 02115, \\ $\mathrm{USA}_{;}{ }^{3}$ State Key Laboratory of Bio-organic and Natural Products Chemistry, Shanghai Institute of Organic Chemistry, Chinese \\ Academy of Sciences, Shanghai 200032, China
}

\begin{abstract}
Aberrant activation of the Wnt signaling pathway plays an important role in human cancer development. Wnt signaling is negatively regulated by Axin, a scaffolding protein that controls a rate-limiting step in the destruction of $\beta$-catenin, the central activator of the Wnt pathway. In Wnt-stimulated cells, Axin is rapidly modified by tankyrasemediated poly(ADP-ribosyl)ation, which promotes the proteolysis of Axin and consequent stabilization of $\beta$-catenin. Thus, regulation of the levels and activity of tankyrases is mechanistically important in controlling Wnt signaling. Here, we identify ubiquitin-specific protease 25 (USP25) as a positive regulator of Wnt/ $\beta$-catenin signaling. We found that USP25 directly interacted with tankyrases to promote their deubiquitination and stabilization. We demonstrated that USP25 deficiency could promote the degradation of tankyrases and consequent stabilization of Axin to antagonize Wnt signaling. We further characterized the interaction between TNKS1 and USP25 by X-ray crystal structure determination. Our results provide important new insights into the molecular mechanism that regulates the turnover of tankyrases and the possibility of targeting the stability of tankyrases by antagonizing their interaction with USP25 to modulate the Wnt/ $\beta$-catenin pathway.
\end{abstract}

[Keywords: tankyrase; USP25; Wnt signaling]

Supplemental material is available for this article.

Received April 23, 2017; revised version accepted May 24, 2017.

The Wnt/ $\beta$-catenin pathway plays a crucial role in development by regulating many cellular processes, including proliferation, cell fate determination, adhesion, polarity, and migration. Hyperactivation of the $\mathrm{Wnt} / \beta$-catenin pathway as a result of mutations in Wnt or $\beta$-catenin itself or truncating mutations in the tumor suppressor APC drives the development of human colon, gastric, and hepatocellular cancers (Polakis 2000; Klaus and Birchmeier 2008). Thus, developing inhibitors of the Wnt/ $\beta$-catenin pathway as a novel anti-cancer therapy has been of great interest in the cancer field (de Sousa et al. 2011; Verkaar and Zaman 2011). One potential target for controlling Wnt/ $\beta$-catenin signaling is Axin (also known as Axin1), a scaffolding protein and an important tumor suppressor that can negatively regulate the $\mathrm{Wnt} / \beta$-catenin pathway by controlling the rate-limiting step mediated by the $\beta$ catenin destruction complex (Luo and Lin 2004; Fearon 2009; MacDonald et al. 2009).

Tankyrase 1 (TNKS1) and TNKS2, members of the PARP family, catalyze the covalent poly-ADP-ribosylation (PARsylation) of target proteins by transferring the ADP-ribose unit from $\beta$-nicotinamide adenine dinucleo-

\footnotetext{
${ }^{4}$ These authors contributed equally to this work.

Corresponding authors: jyuan@hms.harvard.edu, panlf@sioc.ac.cn Article published online ahead of print. Article and publication date are online at http://www.genesdev.org/cgi/doi/10.1101/gad.300889.117.
}

tide $\left(\beta-\mathrm{NAD}^{+}\right)$to substrates (Smith et al. 1998). Tankyrase was originally discovered as a regulator of telomere maintenance in mediating PARsylation of telomeric repeatbinding factor 1 (TRF1) to control its binding to telomeric DNA (Smith et al. 1998; Riffell et al. 2012). In the Wnt/ $\beta$ catenin pathway, tankyrase PARsylates Axin, which in turn promotes its ubiquitination by the ubiquitin E3 ligase RNF146 and the subsequent proteasomal degradation (Huang et al. 2009; Callow et al. 2011; Zhang et al. 2011). Since the stabilization of Axin can lead to a concomitant reduction in $\beta$-catenin protein levels, which blocks its transcriptional activity in vitro and in vivo, inhibition of tankyrases has been proposed as an exciting new cancer therapeutic approach to block Wnt signaling (Huang et al. 2009; Waaler et al. 2011, 2012). However, catalytic inhibition of tankyrases might not be sufficient, as polymers of tankyrases were discovered to act as scaffolds to promote Wnt signaling independently of their PARP activity (Mariotti et al. 2016). Thus, inhibiting the catalytic activity of tankyrases per se may not be adequate

(C) $2017 \mathrm{Xu}$ et al. This article is distributed exclusively by Cold Spring Harbor Laboratory Press for the first six months after the full-issue publication date (see http://genesdev.cshlp.org/site/misc/terms.xhtml). After six months, it is available under a Creative Commons License (Attribution-NonCommercial 4.0 International), as described at http://creativecommons.org/licenses/by-nc/4.0/. 
for suppressing Wnt activity in cells with high levels of tankyrases.

Deubiquitination is an important cellular mechanism that regulates protein stability (Reyes-Turcu et al. 2009). In this study, we show that ubiquitin-specific protease 25 (USP25), a deubiquitinating enzyme (DUB), positively regulates Wnt signaling by mediating the deubiquitination of tankyrases. Deubiquitination of tankyrases by USP25 promotes their stabilization and activation of Wnt signaling. Conversely, USP25 deficiency promotes the degradation of tankyrases and consequently leads to the stabilization of Axin to negatively regulate $\mathrm{Wnt} / \beta$-catenin signaling. We found that the C terminus of USP25 directly interacted with the ankyrin repeats of TNKS1. We further characterized the interaction between TNKS1 and USP25 by X-ray crystal structure determination. Our work provides an important new insight into the molecular mechanism that regulates the turnover of tankyrases and the possibility of targeting the stability of tankyrases to modulate the Wnt/ $\beta$-catenin pathway.

\section{Results}

Identification of tankyrases as preferential USP25-binding proteins

We analyzed the binding proteins of USP2 5 by mass spectrometry and identified both tankyrases, TNKS1 and TNKS2, as the top hits (Fig. 1A; Supplemental Fig. S1A). The interaction between USP25 and TNKS1/2 is confirmed by coimmunoprecipitation assay (Fig. 1B; Supplemental Fig. S1B). Moreover, we observed that Flag-USP25 interacted efficiently with endogenous TNKS1/2 (Fig. 1C).

The interaction of USP25 with TNKS1 is critically dependent on the C-terminal part of USP25, as the expression of the C-terminal 335 amino acid residues was sufficient to support its binding with TNKS1, while the truncation of its C-terminal 337 amino acid residues totally disrupted the interaction between USP25 and TNKS1 (Fig. 1D,E). The N-terminal part of TNKS1, consisting of five ankyrin repeat clusters (ARCs), was necessary and sufficient for the interaction with USP25, whereas the removal of the SAM and PARP domains from its C-terminal part had no effect on its interaction with USP25 (Fig. 1F). We expressed and purified the recombinant C-terminal part of GST-USP25-C (residues 720-1055) and the ankyrin repeat domain of Trx-TNKS1-ANK (residues 178-957). Coincubation of GST-USP25-C and Trx-TNKS1-ANK led to the formation of a larger-molecular-weight complex when analyzed by analytic size exclusion chromatography, suggesting the direct interaction between the C-terminal part of USP25 and the ankyrin repeat domain of TNKS1 (Fig. 1G).

\section{USP25 deubiquitinates and stabilizes tankyrases}

Overexpression of USP25 significantly increased the protein levels of both exogenous and endogenous tankyrases (Fig. 1B,C). Furthermore, we found that ectopic expression of USP25 was able to increase the protein levels of both
TNKS1 and TNKS2 in a dose-dependent manner (Fig. 2A; Supplemental Fig. S2A). To investigate the effect of USP2 5 on the stability of TNKS1/2 protein, we treated control cells or cells expressing USP25 with the protein synthesis inhibitor cycloheximide (CHX) and measured the levels of TNKS1/2 proteins. We found that TNKS $1 / 2$ had a very short half-life $\left(t_{1 / 2}\right)$ normally, as the levels could be significantly reduced upon treatment with $\mathrm{CHX}$ for $3 \mathrm{~h}$; on the other hand, the levels of TNKS1/2 were minimally affected in cells overexpressing USP25 even after prolonged treatment of CHX (Fig. 2B).

To further characterize the role of USP25 in regulating the levels of tankyrases, we generated a loss-of-function allele of USP25 in HEK293T cells using CRISPR-Cas9 technology (Supplemental Fig. S2B). We found that the loss of USP25 led to significant reductions in the protein levels of TNKS1/2 (Fig. 2C). Consistently, the half-life of TNKS1/2 was correspondingly shortened in HEK293T cells with the loss of USP25 (Fig. 2D). Using siRNAs, we further demonstrated that depletion of USP25 decreased the abundance of TNKS1/2 in both DLD-1 and SW480 cells (Fig. 2E). Consistently, USP25 deficiency in DLD-1 cells generated by CRISPR/Cas9-mediated knockout also significantly shortened the half-lives of TNKS1/2 (Fig. 2F). Taken together, these results suggest that USP25 promotes the stability of tankyrases.

Since USP25 is a deubiquitylase, we went on to examine whether its catalytic activity is required to modulate the levels of tankyrases. Indeed, a catalytically inactive C178S USP25 mutant, which could interact with TNKS1/2 normally (Supplemental Fig. S2C), had lost its ability to up-regulate TNKS1/2, compared with wild-type USP25 (Fig. 2G). Thus, the DUB activity of USP25 is critically important for regulating the levels of tankyrases.

We next examined whether USP25 regulated the ubiquitination levels of tankyrases in cells. RNF146 has been reported to be an E3 ubiquitin ligase for tankyrases (Callow et al. 2011; Zhang et al. 2011; DaRosa et al. 2015). The expression of RNF146 induced the ubiquitination of TNKS1/2, which was significantly diminished by coexpression of USP25 (Fig. 2H; Supplemental Fig. S2D). On the other hand, the DUB-inactive C178S USP25 mutant lost the ability to reduce the ubiquitination of TNKS1/2 induced by RNF146 (Fig. 2H). Conversely, the ubiquitination levels of TNKS1/2 were increased in Usp25 $5^{-/-}$cells (Supplemental Fig. S2E). Thus, USP25 negatively regulates the ubiquitination levels of tankyrases in cells.

\section{USP25 binds to the ankyrin repeat region of tankyrases}

The C terminus of USP25, essential for interacting with tankyrases, contains a stretch of sequence (RTPADG) that is highly evolutionarily conserved among USP25 orthologs in difference species (Fig. 3A) and similar to the RXXXDG tankyrase-binding motif (Guettler et al. 2011). Since the N-terminal part of TNKS1, consisting of five ARCs, was necessary and sufficient for the interaction with USP25, we focused our analysis on the possible interaction of ARCs with the C terminus of USP25. We 


\begin{tabular}{|c|cc|}
\hline \multicolumn{1}{|c|}{ Protein } & \multicolumn{2}{|c|}{ Total peptides } \\
\cline { 2 - 3 } & Ctrl & Flag-USP25 \\
\hline USP25 & 12 & 1497 \\
TNKS & 0 & 96 \\
TNKS2 & 0 & 66 \\
KLHL9 & 0 & 35 \\
KLHL13 & 0 & 34 \\
RAD23B & 2 & 29 \\
CUL2 & 0 & 29 \\
CUL3 & 0 & 28 \\
HRNR & 0 & 19 \\
NEDD8 & 0 & 8 \\
KLHDC10 & 0 & 8 \\
MYO6 & 0 & 8 \\
KCTD10 & 0 & 7 \\
UGGT1 & 0 & 6 \\
FEM1B & 0 & 6 \\
SENP2 & 0 & 6 \\
\hline
\end{tabular}

E

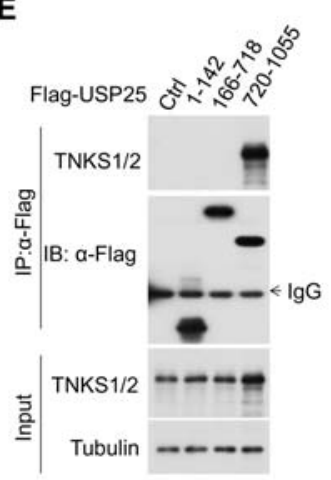

B

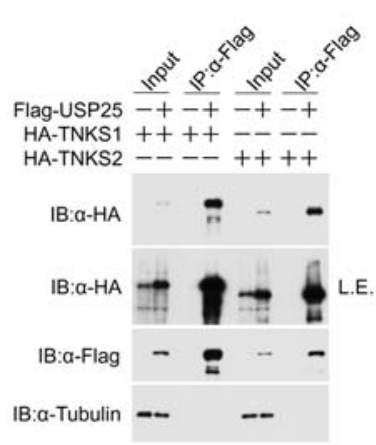

D
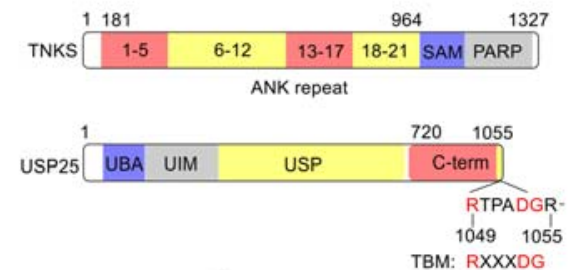

$F$

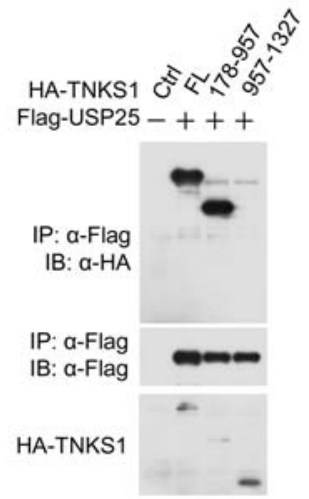

C

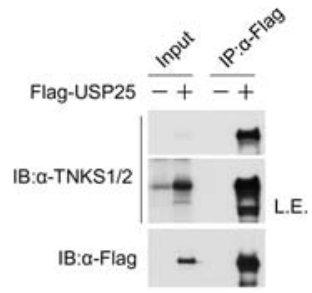

G

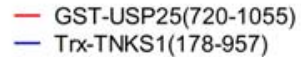

- Mixture

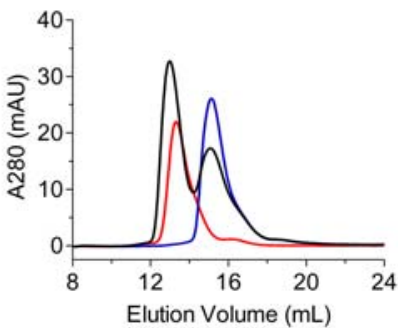

Figure 1. Tankyrases bind preferentially to USP25. (A) A mass spectrometric analysis of proteins that bound with USP25. Flag-USP25 vector was transfected into HEK293T cells, and anti-Flag was used to immunoprecipitate USP25-binding proteins. Anti-Flag immunocomplexes were analyzed by mass spectrometry. The total numbers of peptides identified for each protein are shown. $(B)$ TNKS1 and TNKS2 interact with USP25. HEK293T cells were transfected with plasmids encoding Flag-tagged USP25 together with vector or plasmids encoding HA-tagged TNKS1 or TNKS2. Immunoprecipitation was carried out using anti-Flag antibody and then analyzed by Western blotting using the indicated antibodies. $(C)$ Interaction between transfected Flag-USP25 and endogenous TNKS1/2. Lysates from HEK293T cells expressing Flag-USP25 were subjected to immunoprecipitation/Western analysis using the indicated antibodies. (D) Schematic representation of TNKS1 and USP25. The putative tankyrase-binding motif in the C terminal part of USP25 (RTPADG) is indicated. $(E)$ The C terminus of USP25 is required for binding with tankyrases. Three deletion constructs of USP25 (Flag-tagged) were individually transfected to HEK293T cells. Immunoprecipitation was carried out using anti-Flag antibody and then analyzed by Western blotting using anti-TNKS1/2 or anti-Flag antibody. (F) USP25 binds to ankyrin repeat region of TNKS1. Immunoassay of HEK293T cells transfected with Flag-USP25 and full-length TNKS1 (FL) and three deletion constructs of TNKS1 (HA-tagged) followed by immunoprecipitation with antiFlag antibody and immunoblot analysis with anti-HA or anti-Flag antibody. ( $G$ ) Analytical gel filtration chromatography analysis of the interaction between purified USP25(720-1055) and TNKS1(178-957) protein.

found that recombinant Trx-TNKS1-ANK (residues 178957) purified from Escherichia coli bound to the peptide of the last seven amino acid residues from USP25 with an affinity of $\sim 6.5 \mu \mathrm{M}$ (Fig. 3B). Using analytical gel filtration chromatography assays, we mapped out that all four ankyrin repeat-containing fragments (ARC1, ARC2-3, ARC4, and ARC5 from TNKS1) could interact with this seven-amino-acid peptide from USP25 (Supplemental Fig. S3A). Further quantitative analyses using isothermal titration calorimetry (ITC) revealed that the last ARC (residues 799-957) showed stronger binding $\left(K_{d}=7.1 \pm\right.$
$0.5 \mu \mathrm{M})$ than other ARCs $\left(K_{\mathrm{d}}=57.9 \mu \mathrm{M} \pm 11.8 \mu \mathrm{M}\right.$ for ARC1, $K_{\mathrm{d}}=32.5 \mu \mathrm{M} \pm 4.4 \mu \mathrm{M}$ for ARC2-3, and $K_{\mathrm{d}}=9.3$ $\mu \mathrm{M} \pm 0.4 \mu \mathrm{M}$ for ARC4) (Fig. 3B; Supplemental Fig. S3B, C). Moreover, the binding affinity of a much longer fragment of the USP25 C terminus (residues 720-1055) showed an affinity $\left(K_{\mathrm{d}}=7.9 \pm 0.3 \mu \mathrm{M}\right)$ similar to the last seven-amino-acid peptide (Fig. 3B). Thus, this seven-amino-acid peptide from the very C terminus of USP25 is the primary epitope responsible for interacting with the ankyrin repeat domain in tankyrases. On the other hand, tankyrases may have up to four binding sites in their 
A

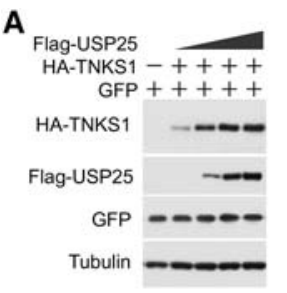

C

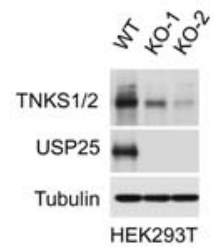

E

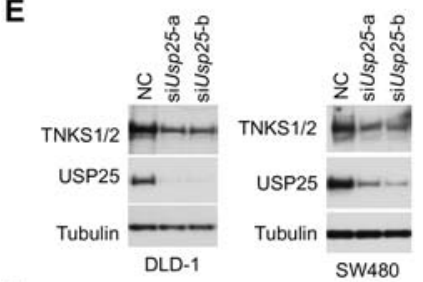

$\mathbf{F}$

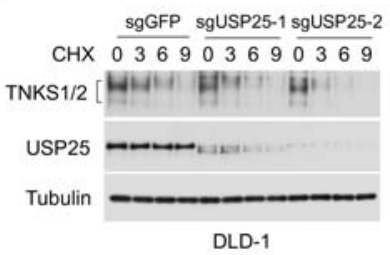

B

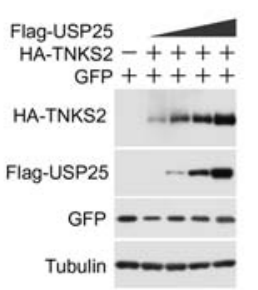

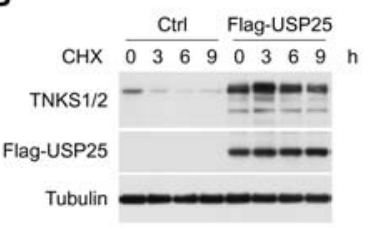

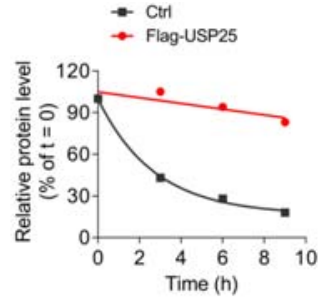

D

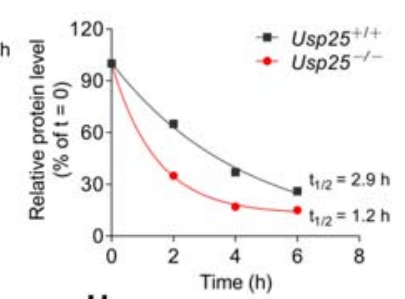

H
G
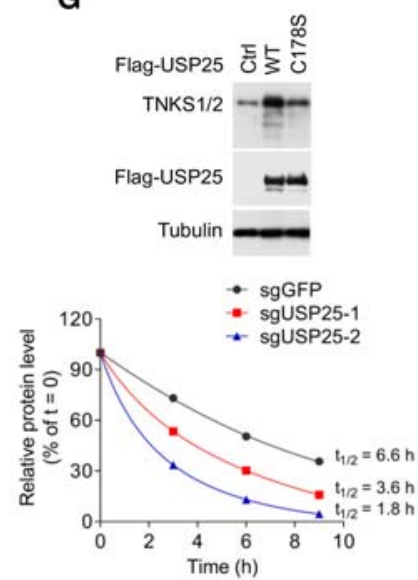

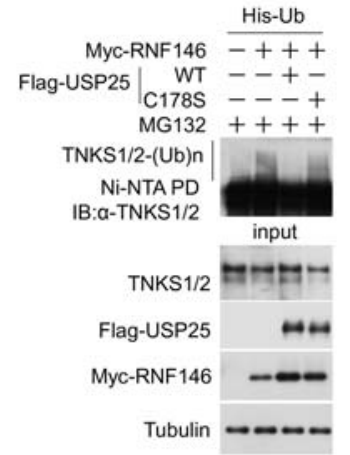

Figure 2. USP25 stabilizes and deubiquitinates tankyrases. (A) USP25 stabilizes both TNKS1 and TNKS2. HA-TNKS1 or HA-TNKS2 was transfected into HEK293T cells with increasing amounts of Flag-USP25, and TNKS1/2 levels were detected. GFP was transfected as a control. $(B)$ USP25 increases the half-life $\left(t_{1 / 2}\right)$ of tankyrases. HEK293T cells transfected with the indicated plasmids were treated with $100 \mu \mathrm{g} / \mathrm{mL}$ CHX and collected at the indicated times for Western blotting. Quantification of tankyrases levels relative to Tubulin is shown. (C) Knockout of USP25 decreases tankyrases. The levels of tankyrases were analyzed in two independent clones of HEK293T cells with USP25 knockout. (D) Half-life analysis of tankyrases in Usp25 $5^{+/+}$and Usp25 ${ }^{-/-}$HEK293T cells. (E) The levels of tankyrases were analyzed in DLD-1 cells and SW480 cells transfected with individual USP25 siRNA. $(F)$ Half-life analysis of tankyrases in DLD-1 cells transduced with Cas9 and single-guide RNAs against USP25. (G) USP25 stabilizes tankyrases in a manner that is dependent on its DUB activity. Expression vectors for USP25 wild type or C178S were transfected into HEK293T cells, and the expression of tankyrases was detected. $(H)$ USP25 deubiquitinates tankyrases. HEK293T cells transfected with the indicated constructs were treated with $10 \mu \mathrm{M}$ MG132 for $6 \mathrm{~h}$ before collection. The whole-cell lysate was subjected to pull-down with $\mathrm{Ni}^{2+}$ beads and $\mathrm{Western}$ blotting with anti-TNKS1/2 antibody to detect ubiquitylated tankyrases.

ankyrin repeat domains for binding to this seven-aminoacid peptide in USP25.

\section{Structural characterization of USP25 and TNKS1 interaction}

To characterize the interaction between USP25 and TNKS1, we determined the crystal structure of TNKS1 ARC5 (residues 799-957) in complex with the USP25 peptide containing its last 10 amino acids (residues 1046-1055) at $1.5 \AA$ resolution (Fig. 3C,D; Supplemental Table 1). The ARC5 contains five ankyrin repeats that stack side by side into a " $\mathrm{C}$ " shape through their a helices packing. The USP25 peptide adopts an extended confor- mation and binds to the central concave side of ARC5 (Fig. 3C,D). The positively charged side chain of R1049 in the RXXXDG motif of USP25 is recognized by a highly negatively charged pocket ("arginine cradle"), where four residues from ARC5 contribute extensive interactions: The side chains of E909 and D900 form two salt bridges with the side chain of R1049, whereas F904 establishes a cation $-\pi$ interaction with the guanidinium group of R1049, and the aromatic ring of W902 packs against the aliphatic side chain of R1049 (Fig. 3C). Interestingly, G1054 is sandwiched by the aromatic side chains of Y880 and Y847 residues in ARC5 through stacking between the backbone of G1054 and the two aromatic rings of Y880 and Y847 (Fig. 3C). These interactions restrict the 


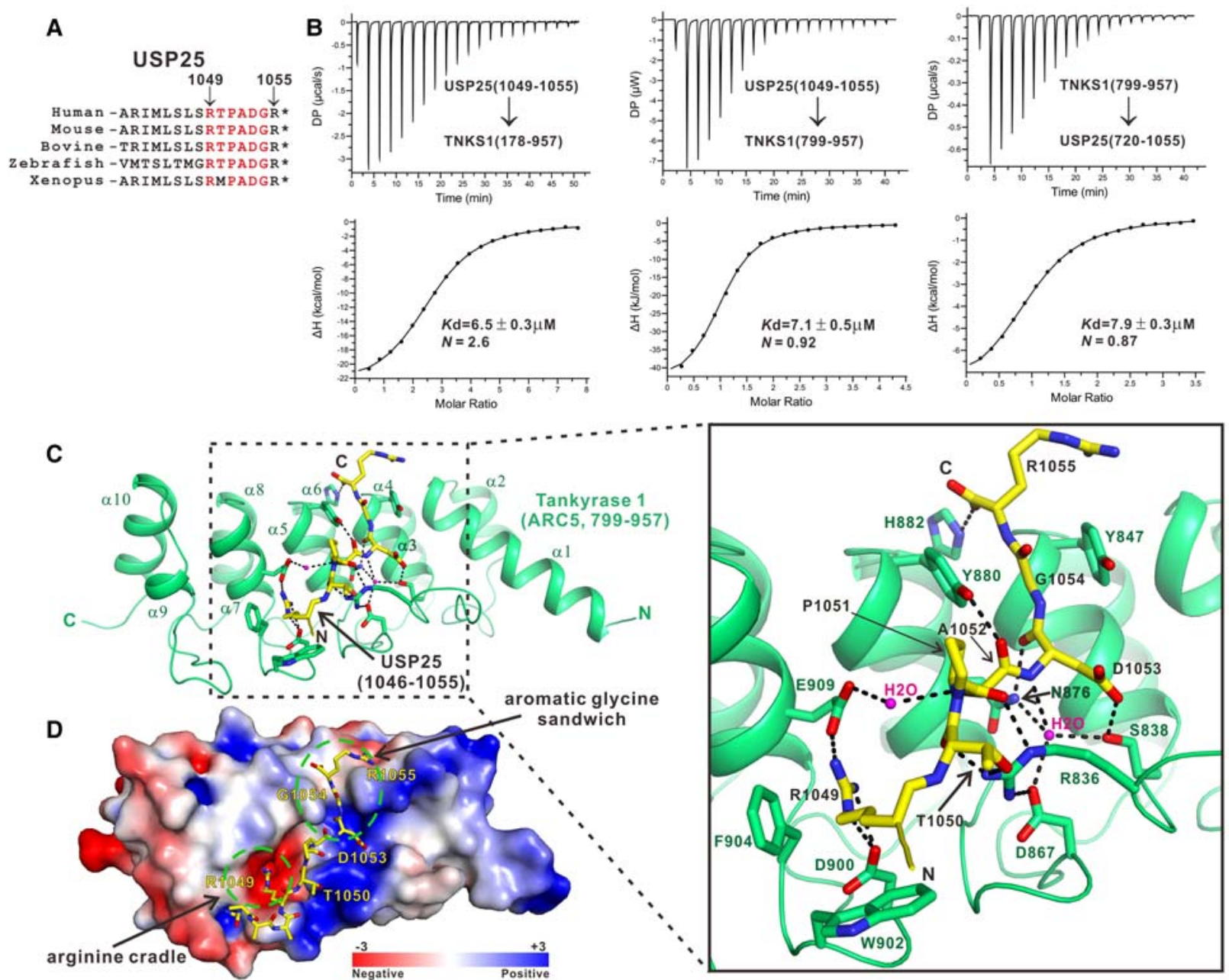

\section{E}

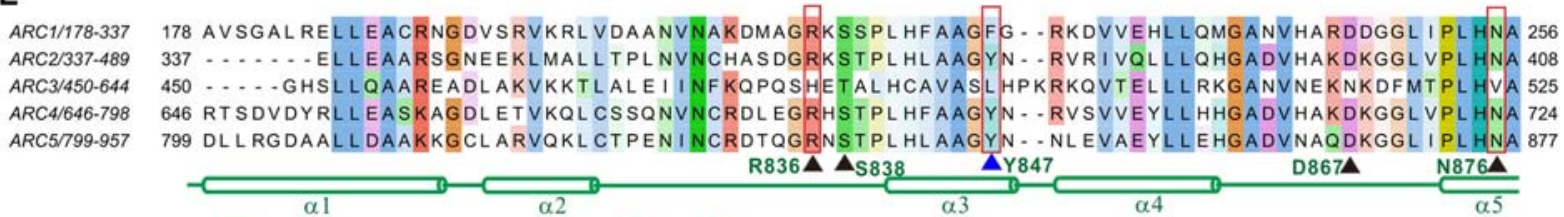
ARC1/178-337 257 CSFGHAEVVSLLL CQGADPNARDNWNYTPLHEAA I KGK I DVCI VLLQHGADPN I RNT DGKSALDLADPSAKAVLTGEYKKD 337 ARC2/337-489 409 CS YGHYEVTELLLKHGACVNAMDLWOFTPL HEAASKNRVEVCSLLLSHGADPTL VNCHGKSAVDMAPTPELRERLTYEFK. 488 ARC3/450-644 526 AERAHNDVMEVL HKHGA KMNALDTLGQTAL HRAALAGHLQT CRLLLSYGSDPS I ISLQGFTAAQMGNEAVQQ IL SESTP. . 604 ARC4/646-798 725 CS YG HYEVAELL VRHGASVNVADLWKFTPL HEAAAKGKYE I CKLLLKHGADPTKKNRDGNTPLDL VKEGDTDIQ....... 798 ARC5/799-957 878 ASYGHVDI AALLI KYNTCVNATDKWAFTPL HEAAQKGRTQL CALLLAHGADPTMKNQEGQTPLDLATADDIRALL I DAMP. 957 -

Figure 3. Biochemical and structural characterizations of the interaction mechanism between TNKS1 and USP25. (A) The highly conserved C termini of USP25 orthologs from different species. (B) ITC assay measuring the binding affinities between the last seven residues (residues 1049-1055) or the C terminus (residues 720-1055) of USP25 and the ankyrin repeat region (residues 178-957) or ARC5 of TNKS1 (residues 799-957) and showing that the last seven residues of USP25 are sufficient for its binding to TNKS1. (C) Crystal structure of the complex formed by TNKS1 ARC5 (residues 799-957) and the last 10 residues of USP25 (residues 1046-1055). The details of interaction are zoomed out in the right panel. The hydrogen bonds and salt bridges are shown as dashed lines. In the crystal structure, two water molecules (shown as magenta balls) are found to intermediate the interaction between USP25 and ARC5. (D) The electrostatic surface shows the "aromatic glycine sandwich" and the charged pockets of TNKS1 ARC5, which accommodate two charged residues (R1049 and D1053) of USP25. The positively or negatively charged surface is colored in blue or red, while neutral is colored in white. (E) Structural-based sequence alignment of five ARCs in TNKS1. The secondary structure of ARC5 is drawn as a cartoon below the alignment. The critical residues in ARC5 interacting with R1049 of USP25 are marked with red triangles, two tyrosine residues (Y847 and Y880) that sandwich G1054 of USP25 are marked with blue triangles, and the residues involved in the recognition of other residues of USP25 are marked with black triangles. Red rectangles mark the positions where ARC3 and other four ARCs show striking differences, indicating the inability of ARC3 to recognize USP25. 
two a hydrogens of G1054, pointing to the bottom of the sandwich, where no space is present to accommodate the side chain of nonglycine residues. Consistently, the substitution of G1054 with alanine that has the smallest side chain (G1054A mutation) completely disrupts the interaction between USP25 and TNKS1/2 (Fig. 4A). The conformation of central residues between R1049 and G1054 is stabilized by an extensive network of polar interactions (Fig. 3C). Particularly, the backbone carbonyl groups of T1050 and P1051 as well as the side chain hy- droxyl group of T1050 form salt bridges with the side chain of TNKS1 R836, which is further coordinated by a salt bridge with D867 in ARC5. In addition, the backbone carbonyl groups of A1052 and D1053 form two hydrogen bonds with the side chains of Y880 and N876, respectively, and the side chain of D1053 forms a hydrogen bond with the side chain hydroxyl group of S838. Meanwhile, the backbone carboxyl group of the extreme C-terminal R1055 residue of USP25 also forms a hydrogen bond with the side chain of H882. In the crystal structure,
A
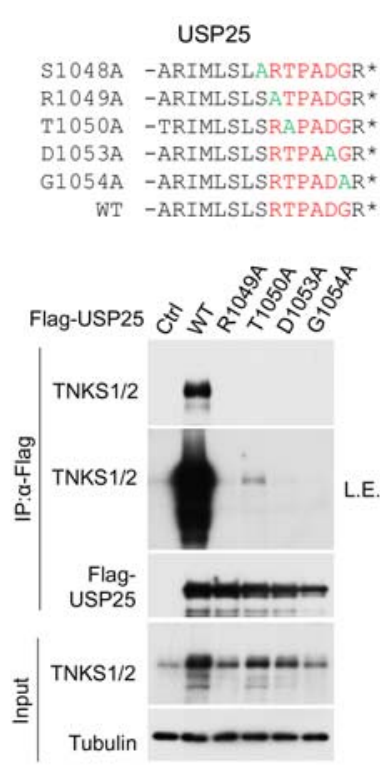

C

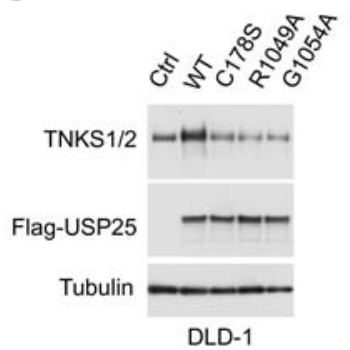

D

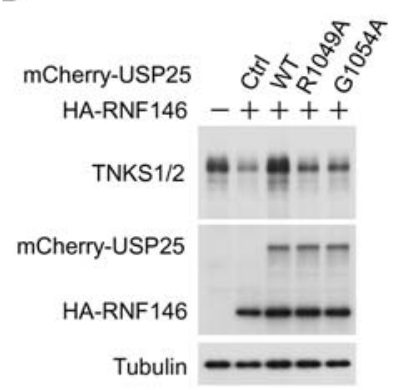

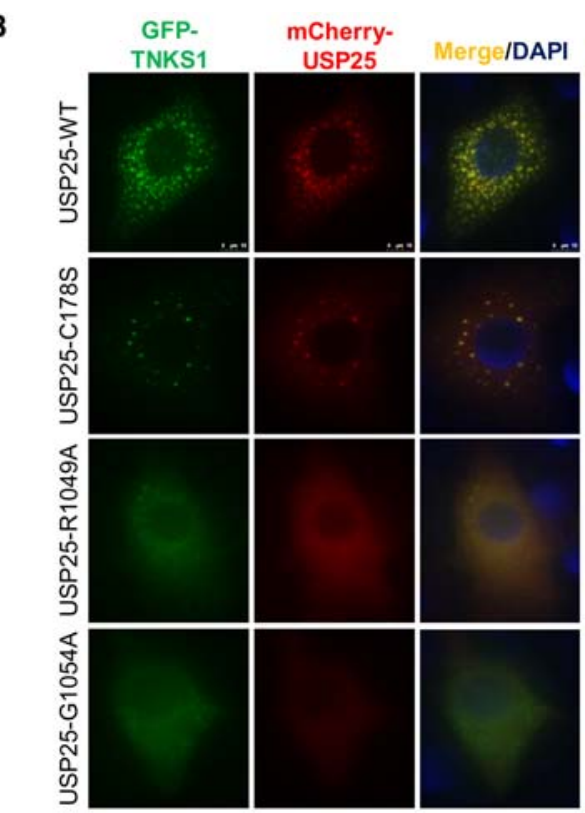

E

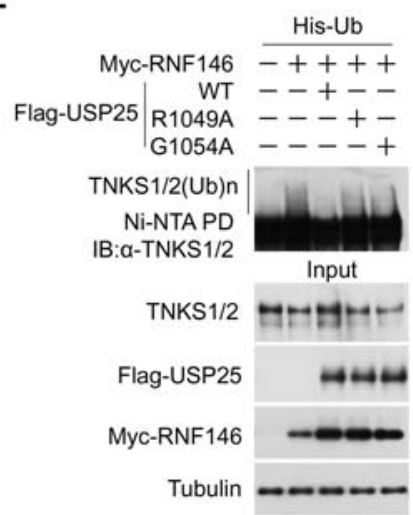

Figure 4. The interaction of USP25 and tankyrases is required for tankyrase stabilization. (A) Mutational analysis of the USP25-tankyrase interaction. The mutations include USP25 S1048A, R1049A, T1050A, D1053A, and G1054A. HEK293T cells were transfected with Flag-tagged wild-type USP25 or mutants as indicated. Lysates were then immunoprecipitated using anti-Flag antibody and immunoblotted using the indicated antibodies. (B) Subcellular localization of USP25 and TNKS1 examined by immunofluorescence. mCherryUSP25 (wild type and mutants) and GFP-TNKS1 were transiently expressed in DLD-1 cells. The cells were then used for immunofluorescence assays. DNA was visualized with DAPI staining. Representative staining is shown. $(C)$ The effect of USP25-tankyrase interaction on tankyrases protein stability. HEK293T cells were transfected with Flag-tagged wild-type or mutant USP25. The protein levels of tankyrases were determined. $(D)$ USP25-tankyrase interaction antagonizes the RNF146-mediated instability of tankyrases. HEK293T cells were cotransfected with Myc-tagged RNF146 and mCherry-tagged wild-type or mutant USP25. The protein levels of tankyrases were determined. $(E)$ The effect of USP25-tankyrase interaction on ubiquitination of tankyrases. HEK293T cells cotransfected with either wildtype or mutant Flag-USP25 and Myc-RNF146 in the presence of His-ubiquitin were treated with $10 \mu \mathrm{M}$ MG132 for 6 h. The whole-cell lysate was subjected to pull-down with $\mathrm{Ni}^{2+}$ beads and Western blotting with anti-TNKS1/2 antibody to detect ubiquitylated tankyrases. 
two water molecules are found to further stabilize this network of polar interaction: One water molecule intermediates the hydrogen bonding between the side chain of E909 and the backbone amide of A1052, while the other water molecule bridges the polar interactions among the side chains of N876, D867, and S838 and the backbone amide group of D1053.

Further structural-based sequence alignment analysis of five ARCs in TNKS1 revealed that all of those critical interface residues are highly conserved in these ARCs except ARC3 (Fig. 3E). For instance, the negatively charged residue E909 in the "arginine cradle" of ARC5 is substituted by a positively charged arginine residue in ARC3, and the crucial aromatic Y880 and Y847 residues in ARC5 are substituted by a leucine and an arginine residue in ARC3, respectively. Furthermore, the residue corresponding to ARC5 N876 that forms a hydrogen bond with the backbone carbonyl group of USP25 D1053 is a hydrophobic valine residue in ARC3. The identification of nonconserved critical interface residues in ARC3 of TNKS1 provides a potential explanation for the inability of ARC3 to recognize the $\mathrm{C}$ terminus of USP25. Thus, we anticipated that the aforementioned ARC2-3 of TNKS1 contained only one USP25-binding site formed by ARC2. Collectively, our structural and sequence analyses clearly demonstrated that the ankyrin repeat region of TNKS1 had four similar binding sites for the C terminus of USP25.

\section{USP25-tankyrase interaction is required for stabilization of tankyrases}

To test our structural observations, we mutated a number of key interacting residues for coimmunoprecipitation assay (Fig. 4A). We ectopically expressed wild-type and mutant Flag-tagged USP25 in HEK293T cells and analyzed the interaction by coimmunoprecipitation with an antiFlag antibody. We found that TNKS1/2 coprecipitated with wild-type USP25 but not with the mutant USP25; namely, R1049A, T1050A, D1053A, and G1054A (Fig. 4A). As a control, mutation S1048A of USP25 had no effect on the association of USP25 and TNKS1/2 (Supplemental Fig. S4A). These results are consistent with the structural data, as R1049A, D1053A, and G1054A mutations completely blocked the interaction of USP25 and TNKS1/2, while the T1050A mutation significantly reduced the association of USP25 with TNKS1/2 (Fig. 4A).

We next investigated the role of the USP25-tankyrase interaction in the cellular localizations of USP25 and tankyrase in DLD-1 cells. When cotransfected, the exogenously expressed mCherry-tagged wild-type USP25 or C178S mutant (a catalytically inactive mutant) showed a puncta staining pattern in the cytoplasm of transfected cells and colocalized well with the GFP-tagged TNKS1 puncta (Fig. 4B; Supplemental Fig. S4B). In contrast, the R1049A and G1054A mutants of USP25 showed a diffused location in transfected cells, and their abilities to colocalize with the GFP-TNKS1 puncta were impaired (Fig. 4B; Supplemental Fig. S4B).

To investigate the functional consequence of USP25tankyrase interaction, we transfected expression vectors for wild-type or mutant USP25 into DLD-1 cells and examined their impact on the levels of TNKS1/2. We found that both R1049A and G1054A mutants were unable to promote the stabilization of TNKS1/2, compared with wild-type USP25 (Fig. 4C). Similar results were also observed in SW480 cells and HCT116 cells (Supplemental Fig. S4C,D). In addition, coexpression of wild-type, but not R1049A and G1054A, USP25 was able to effectively protect the degradation of TNKS1/2 induced by RNF146 (Fig. 4D).

We next performed in vivo ubiquitination assays, monitoring ubiquitination of tankyrases in the presence of either wild-type USP25 or these mutants. As shown in Figure 4E, R1049A and G1054A mutants were not able to mediate deubiquitination of TNKS1/2, compared with wild-type USP25. Taken together, these results suggest that the binding and deubiquitinating activities of USP25 are critically important for its ability to protect tankyrases from degradation.

\section{USP25 modulates the Wnt/ $\beta$-catenin pathway through tankyrases}

Tankyrases have been reported to regulate Wnt signaling by PARsylating Axin to promote its degradation (Huang et al. 2009). Since we found that USP25 could stabilize tankyrases, we reasoned that USP25 might modulate the Wnt pathway through up-regulating tankyrases to promote the degradation of Axin. To test this possibility, we characterized the protein levels of Axin1 in USP25 knockout HEK293T cells generated via CRISPR-Cas9 technology. As shown in Figure 5A, Axin1 levels were increased in USP25 knockout cells, suggesting that USP25 might regulate Wnt signaling.

To further characterize the effect of USP25 on Wnt signaling, we determined the impact of USP25 expression using a well-established Wnt responsive Super-Topflash (STF) luciferase reporter assay in HEK293T cells. We found that knockdown of USP25 significantly reduced this Wnt-driven luciferase activity (Fig. 5B). Consistently, knockdown of USP25 also blocked Wnt3a-induced accumulation of $\beta$-catenin in HEK293T cells (Fig. 5C). Moreover, the Wnt3a-stimulated STF activity and accumulation of $\beta$-catenin were strongly inhibited in Usp25 $5^{-/-}$HEK293T cells as compared with $U s p 25^{+/+}$cells (Fig. 5D,E). However, knockout of USP25 did not affect NF- $\kappa B$ luciferase reporter as reported previously (Supplemental Fig. S5A; Zhong et al. 2012). Finally, USP25 knockout also inhibited the expression of the $\beta$-catenin target gene AXIN2 (Fig. 5F).

It has been reported recently that tankyrase-mediated PARsylation of Axin not only controls its level but also allows PARsylated Axin to directly promote Wnt signaling (Yang et al. 2016). In order to detect whether USP25 affects the levels of PARsylated Axin1 in Wnt stimulation, we used a previously developed pull-down assay based on the ability of the Trp-Trp-Glu (WWE) domain of the RING-type E3 ubiquitin ligase RNF146 to bind to PARsylated proteins (Supplemental Fig. S5B; Callow et al. 2011; Zhang et al. 2011; Yang et al. 2016). Within $2 \mathrm{~h}$ of Wnt3a 

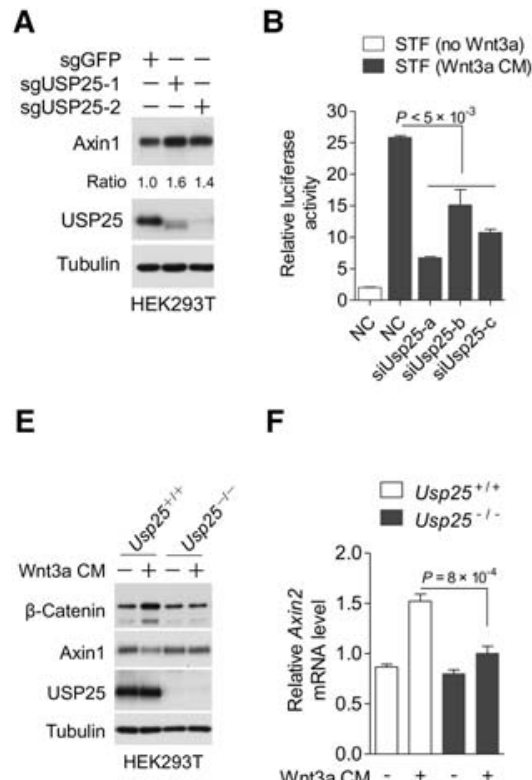

$\mathbf{F}$

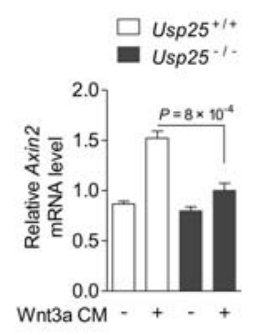

C

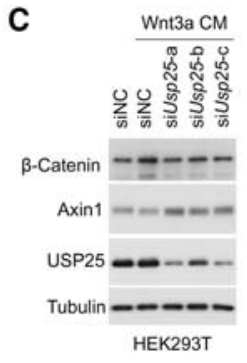

D

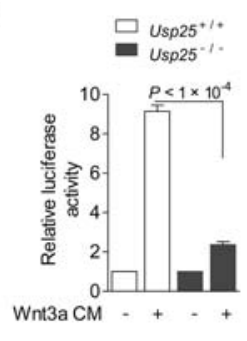

G

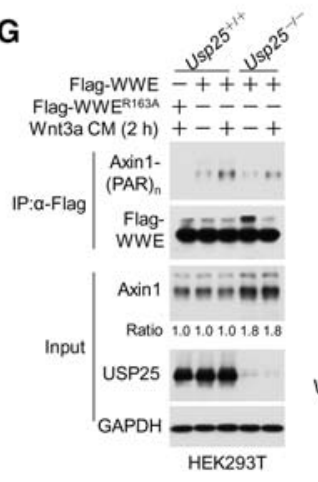

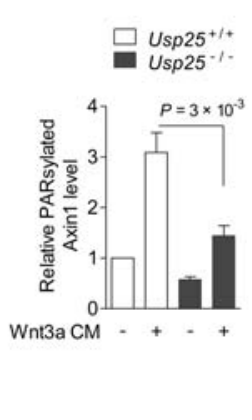

J
H

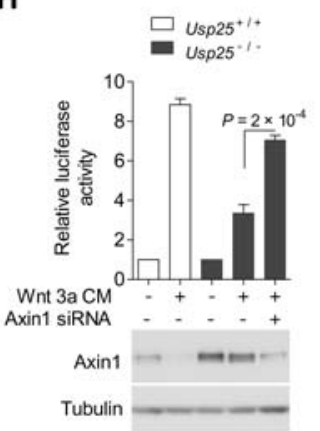

I

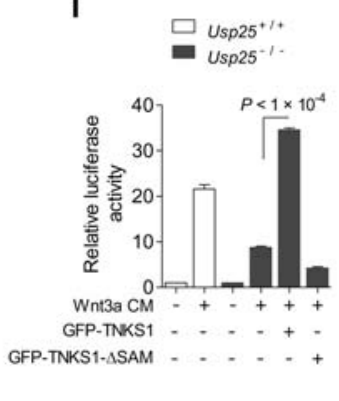

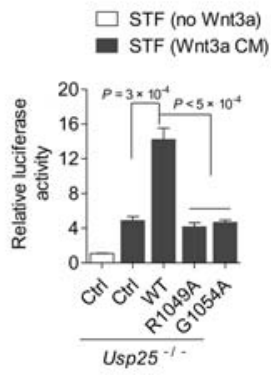

Figure 5. USP25 regulates $\mathrm{Wnt} / \beta$-catenin pathway through tankyrases. $(A)$ Deletion of USP25 increases Axin1 protein levels. HEK293T cells with Lenti-CRISPR-mediated USP25 deletion were harvested and then analyzed by Western blotting using the indicated antibodies. The same results were obtained from three independent sets of experiments. $(B)$ Knockdown of USP25 inhibits the Wnt3a-induced Super-Topflash (STF) reporter in HEK293T cells. Error bars denote the SD between three replicates. (C) Knockdown of USP25 blocks Wnt3a-induced accumulation of $\beta$-catenin in HEK293T cells. HEK293T cells were stimulated with or without Wnt3a for $16 \mathrm{~h}$, and cells were harvested and then analyzed by Western blotting using the indicated antibodies. $(D-F)$ Deletion of USP25 inhibits the Wnt3a-induced STF reporter $(D)$, blocks Wnt3a-induced accumulation of $\beta$-catenin $(E)$, and abolishes Wnt3a-induced Axin2 up-regulation $(F)$ in HEK293T cells. $(G)$ Immunoblot of lysates from HEK293T cells treated with Wnt3a conditioned medium (CM) for $2 \mathrm{~h}$ and then subjected to FlagWWE (Trp-Trp-Glu) pull-down. Treatment with Wnt3a CM increased the level of ADPribosylated Axin pulled down with FlagWWE. Ratios of PARsylated-Axin1/WWE were calculated and are shown at the right. Results are shown as means \pm SD of three independent sets of experiments. $(H)$ The effect of USP25 on Wnt signaling is Axindependent. Usp25 $5^{-1-}$ HEK293T cells were transfected with siRNAs against Axin1 or control siRNA and tested in the STF reporter assay. Knockdown efficiency is shown below. Error bars denote the SD between three replicates. (I) USP25 knockout-mediated inhibition of STF reporter is rescued by the introduction of TNKS1 but not the $\triangle$ SAM mutant. Usp25 $5^{-/-}$HEK293T cells stably expressing GFP-tagged wild-type TNKS1 or the TNKS1- $\triangle$ SAM mutant were tested in the STF reporter assay. Error bars denote the SD between three replicates. (J) The interaction between USP25 and tankyrases is required for USP25-promoted Wnt signaling. Usp25 $5^{-/-}$HEK293T cells reconstituted with Cherry-tagged wild-type USP25 or mutants were tested in the STF reporter assay. Error bars denote the SD between three replicates.

exposure, the levels of PARsylated Axin1 increased as reported previously (Yang et al. 2016), but this increase was notably reduced in Usp25 $5^{-/}$cells (Fig. 5G). Collectively, these results suggest that USP25 is a positive modulator of the $\mathrm{Wnt} / \beta$-catenin pathway.

While knockout of USP25 increased Axin1 protein levels and thus inhibited Wnt signaling, we found that decreasing Axin1 by siRNA-mediated knockdown restored the STF activity in Usp25 $5^{-1-}$ HEK293T cells (Fig. 5H). In addition, introducing wild-type, but not the catalytically inactive SAM deletion mutant of, TNKS1 by lentivirusmediated infection into Usp25 $5^{-/-}$HEK293T cells (Supplemental Fig. S5C) restored STF activity (Fig. 5I; De Rycker and Price 2004). These results indicated that USP25 regulates Wnt signaling via modulation of tankyrase/Axin.

To investigate the effects of the USP25-tankyrase interaction on Wnt signaling, wild-type USP25 as well as the R1049A and G1054A mutants were introduced into Usp25 $5^{-/-}$HEK293T cells individually (Supplemental Fig. S5D), and we found that the expression of wild-type USP25, but not these mutants, restored the STF activity in Usp25 $5^{-/}$HEK293T cells (Fig. 5J), suggesting that USP25 modulates the Wnt pathway through the interaction with tankyrases.

\section{USP25 regulates DLD-1 cell growth in a tankyrase- dependent manner}

Tankyrase is a well-characterized Wnt promoter and is critical for the survival and growth of Wnt-dependent cells, such as DLD-1, a colorectal cancer cell line with mutation in APC (van den Brink et al. 2004). Since USP25 can positively regulate the protein levels of tankyrases, we hypothesized that USP25 might have a proproliferating activity on Wnt-dependent cell lines. We stably knocked out USP2 5 by using the CRISPR-Cas9 technology in these 
cells and found that cells with USP25 knockout proliferated considerably slower than control cells (Fig. 6A). Under low-serum growth conditions, knockout of USP25 significantly inhibited colony formation of DLD1 cells (Fig. 6B).

If the anti-proliferative effect of USP25 knockout on DLD-1 cells was mediated by a reduction in the protein levels of tankyrases, restoration of TNKS1 may rescue the growth inhibitory effects of USP25 knockout. Indeed, restoration of tankyrase expression by introducing a GFPtagged TNKS1 to a level comparable with that of endogenous tankyrases in USP25 knockout cells markedly blocked the anti-proliferative effect of USP25 deficiency, as determined by cell proliferation assay and colony formation assay (Fig. 6C,D). Thus, USP25 promotes the growth of DLD-1 cells in a tankyrase-dependent manner.

Since tankyrases promote Wnt signaling through destabilization of Axin (Huang et al. 2009), we then reasoned that if the anti-proliferative effect of USP25 deficiency in DLD-1 cells was mediated by an increase in the protein levels of Axin, knockdown of Axin $1 / 2$ should block this effect. Indeed, siRNA-mediated depletion of both Axin1 and Axin2 proteins (Huang et al. 2009) completely abolished the anti-growth effect of USP25 knockout (Fig. $6 \mathrm{E})$, supporting the hypothesis that the growth-promoting effects of USP25 in DLD-1 cells were due to Axin-dependent inhibition of Wnt signaling.

To investigate the effect of the USP25-tankyrase interaction on the growth of DLD-1 cells, we introduced either wild-type USP25 or the R1049A mutant individually to USP25 knockout DLD-1 cells, which led to protein expression levels comparable with that of endogenous USP25 (Fig. 6F). Reintroduction of wild-type USP25, but not the R1049A mutant, reversed the reduction in cell growth and colony formation caused by USP25 knockout (Fig. 6F, G), suggesting that the interaction of USP25-tankyrase is critical for DLD-1 cells growth. From these results, we conclude that USP25-mediated stabilization of tankyrases controls the protein levels of Axin to regulate the Wnt/ $\beta$-catenin pathway (Fig. $6 \mathrm{H}$ ).
A

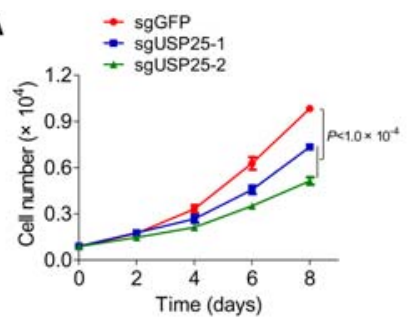

C

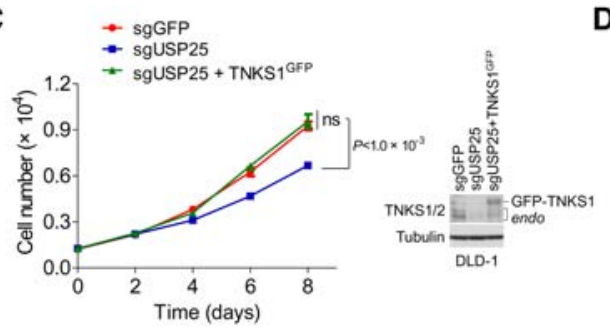

E

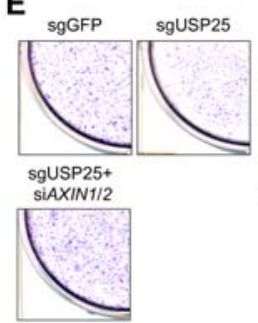

G

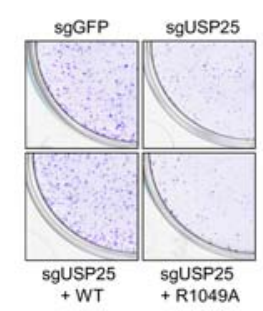

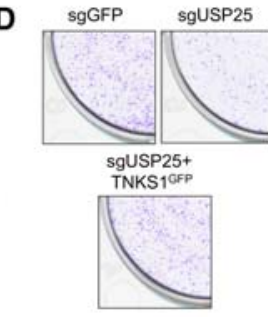

B
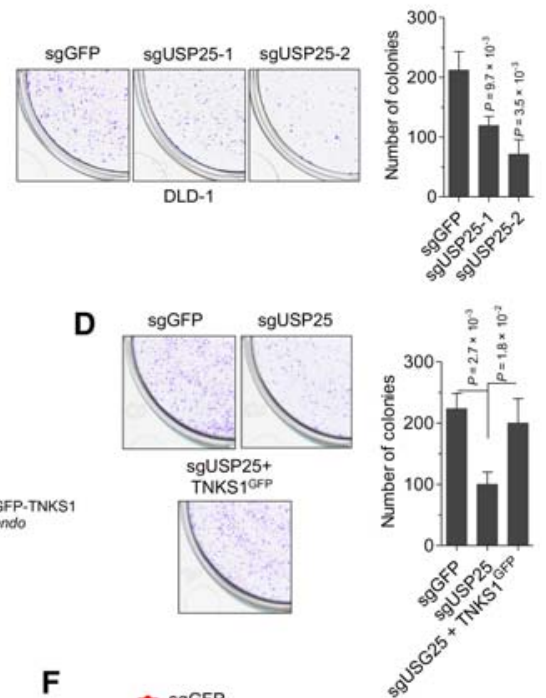

$\mathbf{F}$

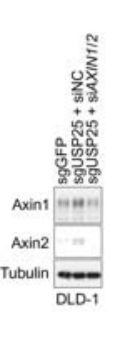

H

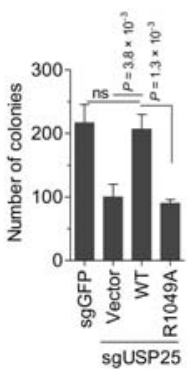

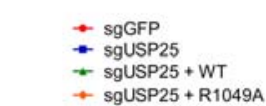
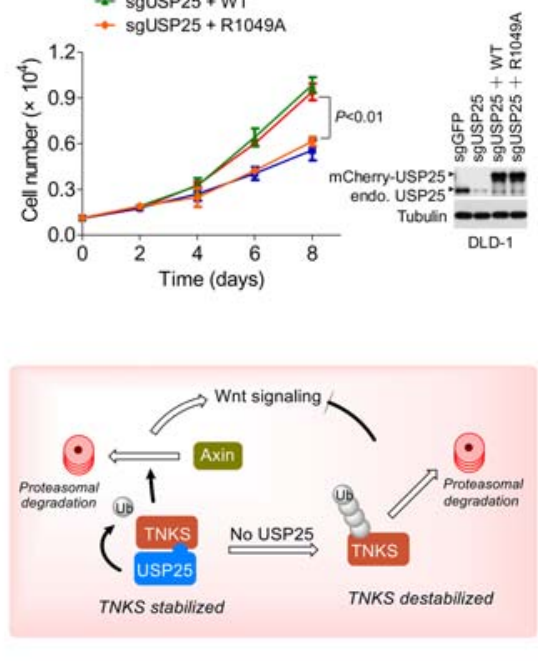

Figure 6. USP 25 regulates cell growth through tankyrases/Axin. $(A, B)$ Deletion of USP25 inhibits the proliferation of DLD-1 cells. DLD-1 cells transduced with sgGFP or two individual single-guide RNAs targeting USP25 were examined to determine their rate of cell proliferation. $(A)$ Cell proliferation assay was performed. Error bars denote the SD between three replicates. $(B)$ Colony formation assay was performed. Colonies viable after 2 wk were counted and analyzed. $n=3$. $(C, D)$ USP25 deletionmediated inhibition of DLD-1 cell growth is rescued by the introduction of GFPtagged TNKS1. $(C)$ Cell proliferation assay was performed. Error bars denote the SD between three replicates. $(D)$ Colony formation assay was performed. Colonies viable after 2 wk were counted and analyzed. $n=$ 3. (E) USP25 deletion-mediated inhibition of DLD-1 cells growth is Axin-dependent. DLD-1 cells were transfected with siRNAs against AXIN1 and AXIN2 or control siRNA and tested in the colony formation assay. $n=3$. Knockdown efficiency was validated by Western blotting and is shown at the right. $(F, G)$ USP25-tankyrase interaction is required for USP25-mediated promotion of DLD-1 cell growth. USP25-deficient DLD- 1 cells generated by the CRISPR-Cas9 system were reconstituted with wild-type USP25 or the R1049A mutant and characterized to determine their rate of cell proliferation $(F)$ and colony formation $(G)$. (H) A working model for USP25-regulated tankyrase stability and Wnt signaling. The deubiquitination of tankyrases by USP25 leads to their stabilization by antagonizing ubiquitination. Stabilized tankyrases promote the degradation of Axin and Wnt signaling. 


\section{Discussion}

In this study, we demonstrate that tankyrases are novel substrates of USP25. We show that the interaction of USP25 and tankyrases is mediated by a conserved last seven-amino-acid peptide in the C terminus of USP25 with the ankyrin repeats of tankyrases. USP25 mediates the deubiquitination of tankyrases to control their stability. Importantly, the expression of USP25 regulates Wnt signaling by controlling the levels of tankyrases and Axin. We propose that USP25 is a crucial positive regulator of Wnt/ $\beta$-catenin signaling.

Our biochemical and structural characterizations of the specific interaction between USP25 and TNKS1 uncover that, except for ARC3, the other four ARCs (ARC1, ARC2, ARC4, and ARC5) of TNKS1 adopt a highly similar binding mode to interact with a consensus RXXXDG motif located at the C terminus of USP25. Particularly, a delicate "arginine cradle" neatly accommodates the R1049 of USP25, an "aromatic glycine sandwich" clamps the G1054 residue, and additional extensive salt bridge and hydrogen bonds further strengthen the association of ARCs to other residues in the motif of USP25. Interestingly, this type of interaction mode is shared by other binders of tankyrases (Guettler et al. 2011). Thus, inhibitors that can disrupt the interaction between USP25 and tankyrases might also block the interaction of tankyrases with other substrates.

Several lines of evidence support the concept that USP25 may function as an oncogene. First, the levels of USP25 mRNAs are significantly increased in breast cancer tissues and non-small cell lung cancer (NSCLC) tissues as compared with adjacent tissues (Deng et al. 2007; Li et al. 2014), although the mechanisms that lead to this up-regulation remain to be elucidated. Second, USP25 can promote cell growth and tumor metastasis. Loss of USP25 was sufficient to decrease tumorigenicity in mammary NSCLC cells. Inhibition of USP25 by miRNA-200c in NSCLC cells significantly inhibited cell migration and metastasis (Li et al. 2014). Since tankyrases have been shown to be involved in three different oncogenic proteins/pathways (Wnt, YAP, and Akt) (Huang et al. 2009; Li et al. 2015; Wang et al. 2015) and since the tankyrase protein levels are significantly up-regulated in human breast cancers (Gelmini et al. 2004), the tumor-promoting role of USP25 may involve the stabilization of tankyrases. Thus, modulating the interaction between USP25 and tankyrases may have effects on additional oncogenic signaling pathways such as those mediated by YAP and Akt.

The interaction of ankyrin repeats in tankyrases with the C terminus of USP25 offers a novel avenue for selectively targeting tankyrases, as ankyrin repeats are not present in other members of PARP family. Modulating the interaction of USP25 with tankyrases provides an opportunity to stabilize Axin by promoting the degradation of tankyrases rather than inhibiting their enzymatic activities. Importantly, our results demonstrate that the interaction of USP25 with tankyrases is defined by a seven-amino-acid peptide, suggesting the possibility of identifying small molecule mimetics to disrupt this inter- action as a novel therapeutic approach for the treatment of not only cancers but also other human diseases with dysregulated Wnt/ $\beta$-catenin activity (Guo et al. 2012).

\section{Materials and methods}

Cell culture, antibodies, and reagents

HEK293T cells were cultured in DMEM (Gibco) with 10\% (v/v) FBS (Gibco) and 1\% penicillin/streptomycin. DLD-1, SW480, and HCT116 cells were maintained in RPMI1640 supplemented with $10 \%(\mathrm{v} / \mathrm{v})$ FBS and $1 \%$ penicillin/streptomycin. Cells were grown in a $37^{\circ} \mathrm{C}$ humidified incubator containing $5 \% \mathrm{CO}_{2}$.

The commercial antibodies used for Western blotting analysis included the following: anti-USP25 (1:1000 dilution; Abcam, ab187156), anti-TNKS1/2 (1:1000 dilution; Santa Cruz Biotechnology, sc-8337), anti-Axin1 (1:1000 dilution; Cell Signaling Technology, no. 2087), anti- $\beta$-catenin (1:1000 dilution; Cell Signaling Technology, no. 9562), anti-Axin2 (1:1000 dilution; Cell Signaling Technology, no. 2151), anti-Flag (1:1000 dilution; Cell Signaling Technology, no. 2368), anti-Myc (1:1000 dilution; Proteintech, 16286-1-AP), anti-HA (1:1000 dilution; Proteintech, 51064-2-AP), and anti-Tubulin (1:10000 dilution; MBL, PM054). Anti-Flag affinity gel (B23101) and anti-HA affinity gel (B23301) were from Biotool, CHX (no. 01810) was from Sigma, and XAV939 (no. S1180) and MG132 (S2619) were from Selleckchem.

Plasmids, siRNA transfection, and luciferase reporter assay

USP25 and its mutants were fused with either mCherry or a Flag epitope at the $\mathrm{N}$ termini and cloned into pcDNA3.1 using Phanta Max Super-Fidelity DNA polymerase (Vazyme Biotech Co., Ltd.) and ClonExpress II cloning kit (Vazyme Biotech Co., Ltd.). The cDNA rescue experiment was done by cloning GFP-tagged TNKS1 or Flag-tagged or mCherry-tagged USP25 into a lentiviral construct. Mutagenesis was performed using MutExpress II mutagenesis kit (Vazyme Biotech Co., Ltd.). Cells were transfected with plasmid DNA using PolyJet DNA in vitro transfection reagent (Signagen Laboratories) according to the manufacturer's instructions. siRNA transient transfections were performed using HiPerFect transfection reagent (Qiagen) according to the manufacturer's instructions. The sequences of siRNAs used in this study were as follows: siUsp25-a (5'-UCGAUGGUGUUCCUA CCUU-3'), siUsp25-b (5'-GGGAGUACUUGAAGGUAAA-3'), siUsp25-c (5'-CACUUCUUGUUGGUACCAA-3'), siTNKS1 (5'CUACAACAGAGUUCGAAUA-3'), siTNKS2 (5'- GAGGGUA UCUCAUUAGGUA-3'), siAXIN1 (5'- GGGCAUAUCUGGAU ACCUG-3'), siAXIN2 (5'- GAGUAGCCAAAGCGAUCUA-3'), and siNC (5'-UUCUCCGAACGUGUCACGUdTdT-3').

For Luciferase reporter assays, HEK293T cells were transfected with STF reporter plasmid and an internal reference plasmid for 4-6 h, and cells were activated with Wnt3a conditioned medium (1:1) for another 12-18 h. STF luciferase assays were performed by using the dual-luciferase assay kit (Promega) according to the manufacturer's instructions.

Lentiviral gene delivery and generation of reconstitution lines

VSV-G pseudotyped lentiviruses were generated by transient transfection of HEK293T cells with pLenti, psPAX2 packaging plasmid, and pMD2.G envelope plasmid in a 4:3:1 ratio. Viral supernatants were collected $48 \mathrm{~h}$ after the transfection. Cleared supernatant was filtered through a 0.45 - $\mu \mathrm{m}$ filter. Polybrene $(8 \mu \mathrm{g} / \mathrm{mL})$ was supplemented to viral supernatants. Lentivirally transduced cells were selected in $1-10 \mu \mathrm{g} / \mathrm{mL}$ puromycin 
for 7 d. Usp25 $5^{-/-}$HEK293T, HEK293T-sgUSP25, and DLD-1sgUSP25 cells were infected with lentiviral particles expressing USP25 (wild type or mutants) or TNKS1 (wild type or mutants). Polyclonal populations were screened until wild-type and mutant lines were generated that had near endogenous USP25 or tankyrases reconstitution levels.

\section{Protein expression and purification}

TNKS1 ARCs and a USP25 (residues 1049-1055) fragment were expressed as Trx-6xHis fusion protein in E. coli BL21 (DE3), cells are disrupted by a high-pressure homogenizer (Shanghai Litu Mechanical Equipment Engineering Co., Ltd., FB-110S) and purified by $\mathrm{Ni}^{2+}$ affinity resin (GE Healthcare). USP25 (residues 720-1072) was fused to an $\mathrm{N}$-terminal GST tag and purified by glutathione affinity resin (GE Healthcare). All proteins for ITC and comigration assay were further purified by size exclusion chromatography on a Superdex $20026 / 60$ column (GE Healthcare) in a buffer containing $20 \mathrm{mM}$ Tris $(\mathrm{pH} 7.5), 100 \mathrm{mM} \mathrm{NaCl}$, and $1 \mathrm{mM}$ DTT.

\section{ITC assay and analytic size exclusion chromatography}

ITC experiments were performed on an ITC200 or PEAQ-ITC machine (Malvern Instruments Ltd.) at $25^{\circ} \mathrm{C}$. The titration data were analyzed using the program Origin 7.0 from MicroCal and fitted using the one-site binding model. Analytic size exclusion chromatography experiments were performed on a Superdex 200 Increase 10/300 column (GE Healthcare). All proteins for these assays were in the buffer containing $20 \mathrm{mM}$ Tris $(\mathrm{pH} 7.5)$, $100 \mathrm{mM} \mathrm{NaCl}$, and $1 \mathrm{mM}$ DTT.

\section{Crystallization and structure determination}

For crystallization, USP25 (1046-1055) was fused to the C-terminal of Trx-6xHis-TNKS1 (799-957) with a linker of TEV recognition sequence (ENLYFQG). The Trx-6xHis tag was cut by protease $3 \mathrm{c}$ and removed by size exclusion chromatography on a Superdex $7526 / 60$ column in a buffer containing $20 \mathrm{mM}$ Tris (pH 7.5), $100 \mathrm{mM} \mathrm{NaCl}, 1 \mathrm{mM}$ DTT, and $1 \mathrm{mM}$ EDTA. Protein was concentrated to $20 \mathrm{mg} / \mathrm{mL}$, digested by TEV protease overnight, and then used to set up sitting drop vapor diffusion crystallization with a reservoir buffer containing $100 \mathrm{mM}$ HEPES $(\mathrm{pH}$ 7.0), $200 \mathrm{mM}$ sodium chloride, and 20\% (w/v) PEG 6000. After $\sim 10 \mathrm{~d}$, crystals were harvested in the reservoir buffer with an additional $25 \%(\mathrm{v} / \mathrm{v})$ glycerol and flash-frozen in liquid nitrogen. Diffraction data sets were collected on the beam line BL19U1 at Shanghai Synchrotron Radiation Facility (SSRF). Phase was obtained by molecular replacement with TNKS2 ARC4 (residues 488-649) structure (Protein Data Bank 3TWX) as a searching template using the software suite PHENIX (Adams et al. 2010). Further structure refinements were performed using COOT and PHENIX (Adams et al. 2010; Emsley et al. 2010).

\section{Cell proliferation and colony formation assays}

For proliferation assays, cells were seeded at a concentration of 1000 cells per well in flat-bottom 96-well microplates. Cell proliferation was measured at the indicated time using Cell Counting Kit-8 (Biotool, B34302) according to the manufacturer's instructions. For colony formation assays, cells were seeded in lowserum growth medium $(0.5 \% \mathrm{FBS})$ at 1000 cells per well in six-well plates in triplicates. Medium was replenished every $3 \mathrm{~d}$, and cells were incubated for $14 \mathrm{~d}$. Resulting colonies were fixed with $4 \%$ PFA and stained by a solution of $2 \mathrm{mg} / \mathrm{mL}$ crystal violet in PBS. The numbers of colonies were counted.

CRISPR-Cas9-mediated gene knockout

Usp25 was knocked out from HEK293T cells using the CRISPR/ Cas9 system, with a guide RNA (gRNA) spanning exon 2 . The gRNA was cloned individually into the pX330 vector and transfected into HEK293T cells. Transfected cells were sorted by fluorescence-activated cell sorting using GFP. Single colonies were screened using Western blot and DNA sequencing to confirm the loss of USP2 5 protein expression. The following target site sequence, transduced via pX330, was used: TCAAGGCTTGCT GTAGTATC (minus strand).

In some of our experiments, effective gene knockdown was achieved via CRISPR/Cas9-mediated genome editing using pLenti-CRISPR v2 (Sanjana et al. 2014). Two target site sequences were cloned into pLenti-CRISPR v2. Lentiviruses were produced and transduced into HEK293T cells and DLD-1 cells via overnight incubation with polybrene. Following medium change and puromycin selection, cells were harvested $7 \mathrm{~d}$ following infection, and gene knockdown was determined by Western blotting. The most effective target guide sequences in terms of knockdown of protein expression by Western blot were chosen for use in our experiments. The following target site sequences, transduced via pLenti-CRISPR v2, were used: sgGFP (TGAACCGCATCGAG CTGAAG; plus strand), sgUSP25-1 (CAAGGCTTGCTGTAGT ATCT; plus strand), and sgUSP25-2 (TGGAAACTTGGAATTA GCAG; plus strand).

\section{Mass spectrometry}

The binding proteins of USP25, obtained by immunoprecipitation against USP25, were trypsin-digested on beads. The peptides were analyzed on an Orbitrap Fusion mass spectrometer (Thermo Scientific). Protein Identification was performed by Thermo Proteome Discoverer (version 1.4) with Sequest HT. The tandem mass spectra were searched against the UniProt human protein database. The precursor mass tolerance was set as $10 \mathrm{ppm}$, and the fragment mass tolerance was set as $0.6 \mathrm{Da}$. The cysteine carbamidomethylation was set as a static modification, and the methionine oxidation was set as a variable modification. The $q$ values of peptide identification were controlled below $1 \%$ using Percolator. The peptide spectrum match values of each protein were used to quantify protein abundances in control and USP25 immunoprecipitation samples.

\section{Cell imaging and statistical analysis}

Cells were fixed with $4 \%$ paraformaldehyde and stained with 3 $\mu \mathrm{g} / \mathrm{mL}$ DAPI. Image data were collected for DAPI-labeled nuclei and GFP/mCherry-tagged intracellular proteins. Student's $t$-test was used for statistical analysis by using GraphPad Prism.

\section{Accession number}

The atomic coordinate of the structure of TNKS1 ARC5 in complex with the tankyrase-binding motif of USP25 has been deposited in the Protein Data Bank (http://www.pdb.org) under ID code 5 GP7.

\section{Acknowledgments}

This work was supported in part by grants from the Chinese Academy of Sciences, the China Ministry of Science and 
Technology Program (2014ZX09102001-002), the China National Natural Science Foundation (31530041), the National Key R\&D Program of China, the National Institute of Neurological Disorders and Stroke (1R01NS082257), and the National Institute on Aging (1R01AG047231) to J.Y.; the National Natural Science Foundation of China (21621002), the Science and Technology Commission of Shanghai Municipality (15JC1400400), and the Strategic Priority Research Program of the Chinese Academy of Sciences (XDB20000000) to L.P.; the Science and Technology Commission of Shanghai Municipality (15ZR1449100) and the China National Natural Science Foundation (31500597) to J.L.; and the Natural Science Foundation of Shanghai (16ZR1443900) to B.S.

\section{References}

Adams PD, Afonine PV, Bunkoczi G, Chen VB, Davis IW, Echols N, Headd JJ, Hung LW, Kapral GJ, Grosse-Kunstleve RW, et al. 2010. PHENIX: a comprehensive Python-based system for macromolecular structure solution. Acta Crystallogr D Biol Crystallogr 66: 213-221.

Callow MG, Tran H, Phu L, Lau T, Lee J, Sandoval WN, Liu PS, Bheddah S, Tao J, Lill JR, et al. 2011. Ubiquitin ligase RNF146 regulates tankyrase and Axin to promote Wnt signaling. PLoS One 6: e22595.

DaRosa PA, Wang Z, Jiang X, Pruneda JN, Cong F, Klevit RE, Xu W. 2015. Allosteric activation of the RNF146 ubiquitin ligase by a poly(ADP-ribosyl)ation signal. Nature 517: 223-226.

Deng S, Zhou H, Xiong R, Lu Y, Yan D, Xing T, Dong L, Tang E, Yang H. 2007. Over-expression of genes and proteins of ubiquitin specific peptidases (USPs) and proteasome subunits (PSs) in breast cancer tissue observed by the methods of RFDD-PCR and proteomics. Breast Cancer Res Treat 104: 21-30.

De Rycker M, Price CM. 2004. Tankyrase polymerization is controlled by its sterile a motif and poly(ADP-ribose) polymerase domains. Mol Cell Biol 24: 9802-9812.

de Sousa EM, Vermeulen L, Richel D, Medema JP. 2011. Targeting Wnt signaling in colon cancer stem cells. Clin Cancer Res 17: 647-653.

Emsley P, Lohkamp B, Scott WG, Cowtan K. 2010. Features and development of Coot. Acta Crystallogr D Biol Crystallogr 66: 486-501.

Fearon ER. 2009. PARsing the phrase 'all in for Axin'-Wnt pathway targets in cancer. Cancer Cell 16: 366-368.

Gelmini S, Poggesi M, Distante V, Bianchi S, Simi L, Luconi M, Raggi CC, Cataliotti L, Pazzagli M, Orlando C. 2004. Tankyrase, a positive regulator of telomere elongation, is over expressed in human breast cancer. Cancer Lett 216: 81-87.

Guettler S, LaRose J, Petsalaki E, Gish G, Scotter A, Pawson T, Rottapel R, Sicheri F. 2011. Structural basis and sequence rules for substrate recognition by tankyrase explain the basis for cherubism disease. Cell 147: 1340-1354.

Guo Y, Xiao L, Sun L, Liu F. 2012. Wnt/ $\beta$-catenin signaling: a promising new target for fibrosis diseases. Physiol Res 61: 337-346.

Huang SM, Mishina YM, Liu S, Cheung A, Stegmeier F, Michaud GA, Charlat O, Wiellette E, Zhang Y, Wiessner S, et al. 2009. Tankyrase inhibition stabilizes axin and antagonizes Wnt signalling. Nature 461: 614-620.

Klaus A, Birchmeier W. 2008. Wnt signalling and its impact on development and cancer. Nat Rev Cancer 8: 387-398.
Li J, Tan Q, Yan M, Liu L, Lin H, Zhao F, Bao G, Kong H, Ge C, Zhang F, et al. 2014. miRNA-200c inhibits invasion and metastasis of human non-small cell lung cancer by directly targeting ubiquitin specific peptidase 25. Mol Cancer 13: 166.

Li N, Zhang Y, Han X, Liang K, Wang J, Feng L, Wang W, Songyang Z, Lin C, Yang L, et al. 2015. Poly-ADP ribosylation of PTEN by tankyrases promotes PTEN degradation and tumor growth. Genes Dev 29: 157-170.

Luo W, Lin SC. 2004. Axin: a master scaffold for multiple signaling pathways. Neurosignals 13: 99-113.

MacDonald BT, Tamai K, He X. 2009. Wnt/ק-catenin signaling: components, mechanisms, and diseases. Dev Cell 17: 9-26.

Mariotti L, Templeton CM, Ranes M, Paracuellos P, Cronin N, Beuron F, Morris E, Guettler S. 2016. Tankyrase requires SAM domain-dependent polymerization to support Wnt- $\beta$ catenin signaling. Mol Cell 63: 498-513.

Polakis P. 2000. Wnt signaling and cancer. Genes Dev 14: 1837-1851.

Reyes-Turcu FE, Ventii KH, Wilkinson KD. 2009. Regulation and cellular roles of ubiquitin-specific deubiquitinating enzymes. Annual Rev Biochem 78: 363-397.

Riffell JL, Lord CJ, Ashworth A. 2012. Tankyrase-targeted therapeutics: expanding opportunities in the PARP family. Nat Rev Drug Discov 11: 923-936.

Sanjana NE, Shalem O, Zhang F. 2014. Improved vectors and genome-wide libraries for CRISPR screening. Nat Methods 11: 783-784.

Smith S, Giriat I, Schmitt A, de Lange T. 1998. Tankyrase, a poly (ADP-ribose) polymerase at human telomeres. Science 282: 1484-1487.

van den Brink GR, Bleuming SA, Hardwick JC, Schepman BL, Offerhaus GJ, Keller JJ, Nielsen C, Gaffield W, van Deventer SJ, Roberts DJ, et al. 2004. Indian Hedgehog is an antagonist of Wnt signaling in colonic epithelial cell differentiation. Nat Genet 36: 277-282.

Verkaar F, Zaman GJ. 2011. New avenues to target Wnt/ $\beta$-catenin signaling. Drug Discov Today 16: 35-41.

Waaler I, Machon O, von Kries JP, Wilson SR, Lundenes E, Wedlich D, Gradl D, Paulsen JE, Machonova O, Dembinski JL, et al. 2011. Novel synthetic antagonists of canonical Wnt signaling inhibit colorectal cancer cell growth. Cancer Res 71: 197-205.

Waaler J, Machon O, Tumova L, Dinh H, Korinek V, Wilson SR, Paulsen JE, Pedersen NM, Eide TJ, Machonova O, et al. 2012. A novel tankyrase inhibitor decreases canonical Wnt signaling in colon carcinoma cells and reduces tumor growth in conditional APC mutant mice. Cancer Res 72: 2822-2832.

Wang W, Li N, Li X, Tran MK, Han X, Chen J. 2015. Tankyrase inhibitors target YAP by stabilizing angiomotin family proteins. Cell Rep 13: 524-532.

Yang E, Tacchelly-Benites O, Wang Z, Randall MP, Tian A, Benchabane H, Freemantle S, Pikielny C, Tolwinski NS, Lee E, et al. 2016. Wnt pathway activation by ADP-ribosylation. Nat Commun 7: 11430.

Zhang Y, Liu S, Mickanin C, Feng Y, Charlat O, Michaud GA, Schirle M, Shi X, Hild M, Bauer A, et al. 2011. RNF146 is a poly(ADP-ribose)-directed E3 ligase that regulates axin degradation and Wnt signalling. Nat Cell Biol 13: 623-629.

Zhong B, Liu X, Wang X, Chang SH, Liu X, Wang A, Reynolds JM, Dong C. 2012. Negative regulation of IL-17-mediated signaling and inflammation by the ubiquitin-specific protease USP25. Nat Immunol 13: 1110-1117. 


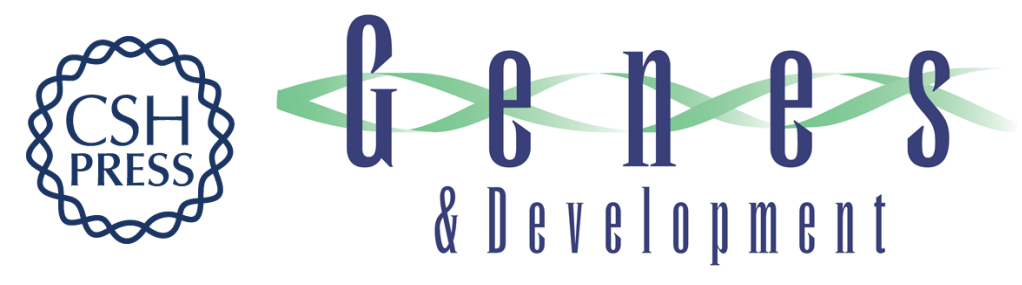

\title{
USP25 regulates Wnt signaling by controlling the stability of tankyrases
}

\author{
Daichao Xu, Jianping Liu, Tao Fu, et al.
}

Genes Dev. 2017, 31: originally published online June 15, 2017

Access the most recent version at doi:10.1101/gad.300889.117

\section{Supplemental http://genesdev.cshlp.org/content/suppl/2017/06/15/gad.300889.117.DC1 Material}

References This article cites 31 articles, 6 of which can be accessed free at: http://genesdev.cshlp.org/content/31/10/1024.full.html\#ref-list-1

Creative This article is distributed exclusively by Cold Spring Harbor Laboratory Press for the first Commons six months after the full-issue publication date (see

License http://genesdev.cshlp.org/site/misc/terms.xhtml). After six months, it is available under a Creative Commons License (Attribution-NonCommercial 4.0 International), as described at http://creativecommons.org/licenses/by-nc/4.0/.

Email Alerting Receive free email alerts when new articles cite this article - sign up in the box at the top Service right corner of the article or click here.

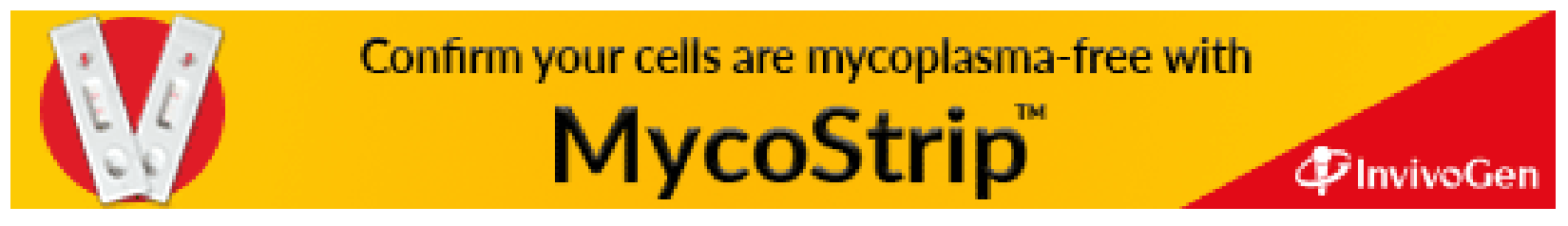

